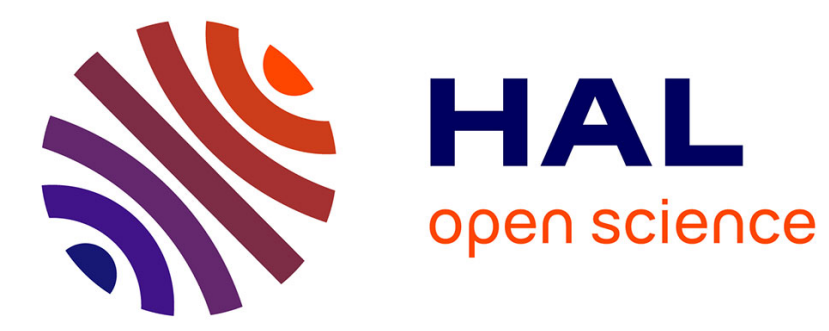

\title{
Ions in an AC Electric Field Strong: Strong long-range repulsion between oppositely charged surfaces
}

Lukasz Richter, Pawel J. Zuk, Piotr Szymczak, Jan Paczesny, Krzysztof M. Bak, Tomasz Szymborski, Piotr Garstecki, Howard A Stone, Robert Holyst, Carlos Drummond

\section{To cite this version:}

Lukasz Richter, Pawel J. Zuk, Piotr Szymczak, Jan Paczesny, Krzysztof M. Bak, et al.. Ions in an AC Electric Field Strong: Strong long-range repulsion between oppositely charged surfaces. Physical Review Letters, 2020, 125, pp.056001. 10.1103/PhysRevLett.125.056001 • hal-02908516

\section{HAL Id: hal-02908516 https://hal.science/hal-02908516}

Submitted on 31 Jul 2020

HAL is a multi-disciplinary open access archive for the deposit and dissemination of scientific research documents, whether they are published or not. The documents may come from teaching and research institutions in France or abroad, or from public or private research centers.
L'archive ouverte pluridisciplinaire HAL, est destinée au dépôt et à la diffusion de documents scientifiques de niveau recherche, publiés ou non, émanant des établissements d'enseignement et de recherche français ou étrangers, des laboratoires publics ou privés. 
archives-ouvertes

\section{Ions in an AC Electric Field: Strong long-range repulsion between oppositely charged surfaces}

Lukasz Richter, Pawel J. Zuk, Piotr Szymczak, Jan Paczesny, Krzysztof M. Bak, Tomasz Szymborski, Piotr Garstecki, Howard Stone, Robert Holyst, Carlos Drummond

\section{To cite this version:}

Lukasz Richter, Pawel J. Zuk, Piotr Szymczak, Jan Paczesny, Krzysztof M. Bak, et al.. Strong longrange repulsion between oppositely charged surfaces. Physical Review Letters, American Physical Society, 2020, 125, pp.056001. 10.1103/PhysRevLett.125.056001 . hal-02908516

\section{HAL Id: hal-02908516 https://hal.archives-ouvertes.fr/hal-02908516}

Submitted on 31 Jul 2020

HAL is a multi-disciplinary open access archive for the deposit and dissemination of scientific research documents, whether they are published or not. The documents may come from teaching and research institutions in France or abroad, or from public or private research centers.
L'archive ouverte pluridisciplinaire HAL, est destinée au dépôt et à la diffusion de documents scientifiques de niveau recherche, publiés ou non, émanant des établissements d'enseignement et de recherche français ou étrangers, des laboratoires publics ou privés. 


\title{
Ions in an AC electric field :
}

\section{Strong long-range repulsion between oppositely charged surfaces}

\author{
Łukasz Richter, ${ }^{1}$ Paweł J. Żuk, ${ }^{2,3}$ Piotr Szymczak, ${ }^{4}$ Jan Paczesny, ${ }^{1}$ \\ Krzysztof M. Bąk, ${ }^{5}$ Tomasz Szymborski, ${ }^{1}$ Piotr Garstecki, ${ }^{1}$ \\ Howard A. Stone, ${ }^{2}$ Robert Hołyst, ${ }^{1, *}$ and Carlos Drummond ${ }^{6,7, \dagger}$ \\ ${ }^{1}$ Institute of Physical Chemistry, Polish Academy of Sciences, \\ Kasprzaka 44/52, 01-224 Warsaw, Poland \\ ${ }^{2}$ Department of Mechanical and Aerospace Engineering, Princeton University, \\ Princeton, New Jersey 08544, USA \\ ${ }^{3}$ Institute of Fundamental Technological Research, Polish Academy of Sciences, \\ Pawińskiego 5b, 02-106 Warsaw, Poland \\ ${ }^{4}$ Faculty of Physics, University of Warsaw, \\ Pasteura 5, 02-106 Warsaw, Poland \\ ${ }^{5}$ Department of Materials, University of Oxford, \\ 21 Banbury Rd, Oxford OX2 6HT, United Kingdom \\ ${ }^{6}$ CNRS, Centre de Recherche Paul Pascal (CRPP), \\ UPR 8641, F-33600 Pessac, France \\ ${ }^{7}$ Université de Bordeaux, CRPP, \\ UPR 8641, F-33600 Pessac, France
}

(Dated: December 12, 2019) 


\begin{abstract}
Two oppositely charged surfaces separated by a dielectric medium attract each other. In contrast we observe a strong repulsion between two plates of a capacitor that is filled with an aqueous electrolyte upon application of an alternating potential difference between the plates. This longrange force increases with the ratio of diffusion coefficients of the ions in the medium and reaches steady state after a few minutes, which is much larger than the millisecond time scale of diffusion across the narrow gap. The repulsive force, an order of magnitude stronger than the electrostatic attraction observed in the same setup in air, results from the increase in osmotic pressure as a consequence of the field-induced excess of cations and anions due to lateral transport from adjacent reservoirs.
\end{abstract}

Manifold electrokinetic phenomena, such as electrohydrodynamic colloid manipulation [1, 2], AC pumping [3], AC electro-osmosis (ACEO) [4], induced-charge electro-osmosis (ICEO) [5], induced-charge electrophoresis (ICEP) [6] and asymmetric rectified electric field (AREF) [7] have been studied experimentally and theoretically [8, 9]. To describe some of these phenomena classical models, like the Poisson-Boltzmann equation, have been modified and expanded, resulting in additional terms to account for large voltages [10, 11], finite sizes of ions [12] and molecular crowding [13], and allows understanding many problems qualitatively and quantitatively.

We present a new phenomenon that eludes all previously developed electrokinetic descriptions - the strong, long-ranged repulsion between surfaces upon application of an AC voltage. Although repulsion of oppositely charged surfaces was reported previously, all such cases were strictly limited to unequal amounts of charges accumulated at both surfaces [14], short-range interactions (compared with the Debye length) [15], multivalent ions [16, 17], exhibiting forces in the range of $\mathrm{nN}$ [16], or ionic liquids [18]. None of these features apply to our measurements.

We utilize a surface forces apparatus (SFA) - a technique for precise measurement of surface interactions [19-22], in which two back-silvered, molecularly smooth mica surfaces are aligned in a crossed-cylinder geometry [20]. One of the surfaces is mounted on a spring, which is deflected in the presence of a normal force (Fig. 1(a)). The separation between the surfaces $H$ is measured by Multiple Beam Interferometry (MBI) $[23,24]$ and its changes (in the absence of a displacement of the distal end of the spring) signals a change in the force 
acting between the surfaces.

The space between the surfaces is filled with an electrolyte and an AC square voltage $V$ is applied at frequencies ranging from $20 \mathrm{~Hz}$ to $500 \mathrm{kHz}$ (Fig. 1(b)). We typically used salt concentration of $1 \mathrm{mM}$, applied AC voltage of $10 \mathrm{~V}$, frequency of $100 \mathrm{kHz}$, and initial separation of $300 \mathrm{~nm}$. Intuitively, application of an AC voltage, which results in oppositely charged surfaces at every time instant, should cause surface attraction. Indeed, in the control measurement we applied the same protocol to the system filled with air and observed an attractive force compatible with the predictions of the classic electrostatic theory (see Eq. 87 Ref. [25]). On the contrary, for case of the capacitor filled with an electrolyte solution, a different behavior was observed: immediately after the application of the AC voltage, the surfaces were electrostatically attracted to each other as the result of a non-vanishing electric field $E$ - similar to [7] - and after approximately 30 seconds strong repulsion appeared (Fig. 1(c)). The time scale at which the separation of the surfaces grew $\tau_{\text {exp }}$ [25] was on the order of few minutes, which is at least five orders of magnitude longer than diffusive charge relaxation time (with diffusion coefficient $D$ ) at the length scale of the gap $\tau_{H}=H^{2} / D$ (milliseconds cf. Table S1 Ref. [25]). The final force was up to $300 \mu \mathrm{N}$ at $10 \mathrm{~V}$ applied potential difference. After the voltage was turned off, the surfaces relaxed to the starting position again on the time scale of minutes. This time is significantly longer than the expected $10 \mathrm{~ms}$ relaxation time of the spring-mass system (see Eq. 83 Ref. [25]). Thus, any previously reported electrokinetic phenomena cannot be the primary reason for the observed repulsion.

We have developed a simplified model to describe the measured repulsion. For this purpose, we identified four distinct length scales present in the experiment of which three are known prior to experiment: the radius of curvature of the surfaces $a=2 \mathrm{~cm}$, the separation between the surfaces $H$ and the Debye length $\lambda_{D}=\sqrt{\epsilon k_{B} T /\left(2 n_{0} e^{2}\right)}$ that depends on bulk salt concentration $n_{0}$, temperature $T$, elementary charge $e$, dielectric constant $\epsilon$ and Boltzmann constant $k_{B}$. Fourth, the distance $d=\sqrt{D \tau_{\text {exp }}}$ of diffusive relaxation during the force buildup is determined during the experiment. Since the Debye length (typically $10 \mathrm{~nm})$ is much smaller than any other dimension $\left(\lambda_{D} \ll H \ll a, \lambda_{D} \ll d\right)$, we represent the two surfaces in contact with the ionic reservoir as a 1-dimensional capacitor of size $L$ (Fig. $1(\mathrm{~d})$ ). We distinguish between $H$, which is the experimental minimal distance between two curved mica surfaces and $L$, which represents size of 1-dimensional capacitor 
in the theoretical model. The model capacitor has one impenetrable electrode connected to an oscillatory potential and a second grounded electrode permeable to ion flux, which is in contact with the ion reservoir. Under these conditions we use the Poisson-Nernst-Planck (PNP) electrokinetic model [25] inspired by earlier works [26-28]. Instead of solving the equations numerically, we have pursued an analytical solution revealing features that do not average out in time. The most important observation is that upon the application of the AC voltage, both cations and anions accumulate on average in the vicinity of the solid electrode (Fig. 1(d)) in contrast to the case of DC voltage where only counterions accumulate at the electrode. The excess of both ions at the surfaces is the element that leads to the difference between the ion concentration inside the capacitor and in bulk reservoir surrounding the device, which creates an osmotic driving force for transport into the capacitor. This dynamic charged double layer has further interesting properties. First, it decays exponentially on the Debye length and vanishes completely at $\sqrt{\lambda_{D} L}$. Second, the magnitude of the ion excess is proportional to $V^{2}$ and, for a large span of $L>\lambda_{D}$, is inversely proportional to $L^{2}$ (Fig. S5(c) Ref. [25]), which means that it is proportional to $E^{2}=(V / L)^{2}$. Third, both cations and anions, on average, accumulate on the oscillating electrode, but there is a larger excess of ions of higher $D\left(\propto V^{2}\right)$. Thus, the layer has a net charge of the more mobile ions (Fig. S6 Ref. [25]). If the repulsion was to come from the direct non-vanishing charge-charge interactions it would scale as $V^{4}$.

We performed the measurements multiple times in the same solution and found that the effect was reversible and reproducible - no degeneration was observed. In agreement with the model, we found that other factors being equal, the force is proportional to the square of the applied voltage (Fig. 2(a)). Moreover, the forcing frequency (up to $500 \mathrm{kHz}$ ) in all experiments falls below the Debye frequency $D / \lambda_{D}^{2}$ (ca. $10 \mathrm{MHz}$ ), which is an important microscopic time scale (cf. Fig. S4 and Table S1 Ref. [25]). In such conditions, the ions are able to adapt their distribution to the changes of the electric field and there is no significant influence of the frequency on the measurements (Fig. S10 Ref. [25]).

We observe the field-induced repulsion for separations from 300 to $2600 \mathrm{~nm}$ (Fig. 2(b)), which is more than two orders of magnitude larger than the Debye length $(\approx 10 \mathrm{~nm})$. Previously reported "long-range" forces in ionic solutions act over distances only few times the Debye length [29-31]. The repulsion does not depend on the separation distance between the surfaces $H$ and similar forces were measured for all initial separations. Using the model 
of the three-layer parallel plate capacitor (Eq. 90 Ref. [25]) for a mica-fluid-mica configuration one can show that the magnitude of the electric field inside the electrolyte is nearly independent of separations, which explains the lack of dependence on $H$ (Fig. S12 Ref. $[25])$.

Previous studies showed that many electrokinetic phenomena arise from differences in ionic diffusion coefficients [15, 32-34]. Thus, we tested 10 electrolytes with ions having even up to tenfold differences in $D$ (Table S1 Ref. [25]) and report results for the force in Fig. 2(c). The measured repulsion increased for larger differences of diffusion coefficient and among tested salts the force varied at most by a factor of nine. In Fig. 3(a) we present the relation between the force measured for different salts and the theoretical prediction of the 1D model of the excess ions at the impenetrable surface, i.e., $F / V^{2}=B n_{\text {exc }}$ (see Eq. 84 Ref. [25]). The only free parameter is the size of the 1D model, $L=2 \mu \mathrm{m}$, which we choose by minimizing the fitting error for $B$; all other parameters correspond to experimental values. For all the salts a good correspondence with the theoretical prediction is obtained.

The differences in $D$ for different salts influence only weakly the time scale at which the repulsion is established. In Fig. 3(b) we show time constants of repulsion for various electrolytes (red bars), which are all around 3.9 \pm 0.8 minutes. Experiments performed for a longer time confirmed that the measured repulsion reaches a steady value (Fig. S13 Ref. [25]). This salt-independent, long characteristic time suggests that the dynamic process is governed by solvent properties. The time scale of minutes is much longer than milliseconds of diffusive relaxation time at the length scale of the gap $\tau_{H}=H^{2} / D$ (Fig. 3(c)). During the $\tau_{\text {exp }}$ the solute and water $\left(D=2.6 \cdot 10^{-9} \mathrm{~m}^{2} / \mathrm{s}[35]\right)$ diffuse over the length $d \approx 800$ $\mu \mathrm{m}$ similar to $\sqrt{a H}$ over which the narrow gap changes significantly. Over this distance the osmotic pressure builds up the repulsion force.

With the proportionality constant $B$ obtained in Fig. 3(a), we compare the theoretical predictions and the experimental measurements for different $\mathrm{NaNO}_{3}$ salt concentrations (Fig. 3(d)). For low to moderate concentrations, the 1D model predictions agree reasonably well with the observed experimental results. According to our model, increasing $n_{0}$ has a two-fold effect: it decreases the Debye length as $\lambda_{D} \propto n_{0}^{-1 / 2}$ (Fig. S5(c) Ref. [25]) and increases the Debye frequency $\omega_{D} \propto \lambda_{D}^{-2}$ (Fig. S5(d) Ref. [25]). These features, when combined together, result in an increase of the excess ion concentration. We performed control measurements in deionized water, where $n_{0}$ was in the range of $0.2 \mu \mathrm{M}$ and the 
measured force was negligible (Fig. S8 Ref. [25]). For very high bulk concentration we expect electrostatic effects to be screened out and the PNP description is no longer valid. As result there should be no difference in concentrations between the bulk and the center of the SFA, i.e. the field-induced osmotic force will vanish. Indeed beyond $10 \mathrm{mM}$ adding salt reduces the repulsion (Fig. 3(d)).

In summary, we have found the emergence of a strong long-range repulsion between curved mica surfaces containing an electrolyte solution upon application of an AC voltage. The strength, range and time scale of the effect exceed by orders of magnitude the values typical of any previous experimental or theoretical work. We proved with theoretical analysis that this effect is of osmotic origin, caused by the accumulation of ions on both electrodes, which triggers the influx of water and ions from a bulk reservoir towards the center of the capacitor. Recently existence of similar effect was reported for ionic liquids [18], that extends applicability of our results. Reported phenomenon can potentially open new paths of utilization of the electric field in such applications as macroscopic separation of charges, artificial ionic pumps, desalination of water, permselective channels, low friction bearings, ionic valves, actuators and tunable capacitors.

Ł.R. acknowledges the support of the National Science Centre, Poland, within the grant Preludium UMO-2017/27/N/ST4/02353. P.J.Z. was supported by the Polish Ministry of Science and Higher Education ("Mobility Plus" Project No. 1294/MOB/IV/2015/0). The work of R.H. was supported by the National Science Centre, Poland, within the grant Maestro UMO-2016/22/A/ST4/00017.

Ł.R. and P.J.Z. equally contributed.

* rholyst@ichf.edu.pl

$\dagger$ drummond@crpp-bordeaux.cnrs.fr

[1] S. C. Bukosky and W. D. Ristenpart, Simultaneous aggregation and height bifurcation of colloidal particles near electrodes in oscillatory electric fields, Langmuir 31, 9742 (2015).

[2] T. J. Woehl, B. J. Chen, K. L. Heatley, N. H. Talken, S. C. Bukosky, C. S. Dutcher, and W. D. Ristenpart, Bifurcation in the steady-state height of colloidal particles near an electrode in oscillatory electric fields: Evidence for a tertiary potential minimum, Phys. Rev. X 5, 011023 
(2015).

[3] A. Ajdari, Pumping liquids using asymmetric electrode arrays, Phys. Rev. E 61, R45 (2000).

[4] A. Ramos, H. Morgan, N. G. Green, and A. Castellanos, Ac electric-field-induced fluid flow in microelectrodes, J. Colloid. Interf. Sci. 217 (1999).

[5] M. Z. Bazant and T. M. Squires, Induced-charge electrokinetic phenomena: theory and microfluidic applications, Phys. Rev. Lett. 92, 066101 (2004).

[6] O. D. Velev, S. Gangwal, and D. N. Petsev, Particle-localized ac and dc manipulation and electrokinetics, Annu. Rep. Prog. Chem., Sect. C: Phys. Chem. 105, 213 (2009).

[7] S. M. H. Hashemi Amrei, S. C. Bukosky, S. P. Rader, W. D. Ristenpart, and G. H. Miller, Oscillating electric fields in liquids create a long-range steady field, Phys. Rev. Lett. 121, 185504 (2018).

[8] M. Z. Bazant, M. S. Kilic, B. D. Storey, and A. Ajdari, Towards an understanding of inducedcharge electrokinetics at large applied voltages in concentrated solutions, Adv. Colloid Interfac. 152, 48 (2009).

[9] M. Z. Bazant and T. M. Squires, Induced-charge electrokinetic phenomena, Curr. Opin. Colloid In. 15, $203(2010)$.

[10] L. Højgaard Olesen, M. Z. Bazant, and H. Bruus, Strongly nonlinear dynamics of electrolytes in large ac voltages, Phys. Rev. E 82, 011501 (2010).

[11] M. Z. Bazant, M. S. Kilic, B. D. Storey, and A. Ajdari, Nonlinear electrokinetics at large voltages, New J. Phys. 11, 075016 (2009).

[12] I. Borukhov, D. Andelman, and H. Orland, Steric effects in electrolytes: A modified poissonboltzmann equation, Phys. Rev. Lett. 79, 435 (1997).

[13] M. S. Kilic, M. Z. Bazant, and A. Ajdari, Steric effects in the dynamics of electrolytes at large applied voltages. i. double-layer charging, Phys. Rev. E 75, 021502 (2007).

[14] V. A. Parsegian and D. Gingell, On the electrostatic interaction across a salt solution between two bodies bearing unequal charges, Biophys. J. 12, 1192 (1972).

[15] H. Aranda-Espinoza, Y. Chen, N. Dan, T. C. Lubensky, P. Nelson, L. Ramos, and D. A. Weitz, Electrostatic repulsion of positively charged vesicles and negatively charged objects, Science 285, 394 (1999).

[16] K. Besteman, M. A. G. Zevenbergen, H. A. Heering, and S. G. Lemay, Direct observation of charge inversion by multivalent ions as a universal electrostatic phenomenon, Phys. Rev. Lett. 
93, $170802(2004)$.

[17] M. Trulsson, B. Jönsson, T. Åkesson, J. Forsman, and C. Labbez, Repulsion between oppositely charged surfaces in multivalent electrolytes, Phys. Rev. Lett. 97, 068302 (2006).

[18] C. S. Perez-Martinez and S. Perkin, Surface forces generated by the action of electric fields across liquid films, Soft Matter 15, 4255 (2019).

[19] J. Israelachvili, Y. Min, M. Akbulut, A. Alig, G. Carver, W. Greene, K. Kristiansen, E. Meyer, N. Pesika, K. Rosenberg, et al., Recent advances in the surface forces apparatus (sfa) technique, Rep. Prog. Phys. 73, 036601 (2010).

[20] C. Drummond, Electric-field-induced friction reduction and control, Phys. Rev. Lett. 109, $154302(2012)$.

[21] K. Kristiansen, H. Zeng, B. Zappone, and J. N. Israelachvili, Simultaneous measurements of molecular forces and electro-optical properties of a confined $5 \mathrm{cb}$ liquid crystal film using a surface forces apparatus, Langmuir 31, 3965 (2015).

[22] R. Tivony, D. B. Yaakov, G. Silbert, and J. Klein, Direct observation of confinement-induced charge inversion at a metal surface, Langmuir 31, 12845 (2015).

[23] J. Israelachvili, Thin film studies using multiple-beam interferometry, J. Colloid Interf. Sci. 44, 259 (1973).

[24] M. Heuberger, The extended surface forces apparatus. Part I. Fast spectral correlation interferometry, Rev. Sc. Instrum. 72, 1700 (2001).

[25] See Supplemental Material at [URL will be inserted by publisher] for the detailed description of experimental, theoretical and numerical methods.

[26] O. Schnitzer and E. Yariv, Nonlinear oscillations in an electrolyte solution under ac voltage, Phys. Rev. E 89, 032302 (2014).

[27] A. Bandopadhyay, V. A. Shaik, and S. Chakraborty, Effects of finite ionic size and solvent polarization on the dynamics of electrolytes probed through harmonic disturbances, Phys. Rev. E 91, 042307 (2015).

[28] R. F. Stout and A. S. Khair, Moderately nonlinear diffuse-charge dynamics under an ac voltage, Phys. Rev. E 92, 032305 (2015).

[29] G. Silbert, D. Ben-Yaakov, Y. Dror, S. Perkin, N. Kampf, and J. Klein, Long-ranged attraction between disordered heterogeneous surfaces, Phys. Rev. Lett. 109, 168305 (2012).

[30] M. A. Gebbie, H. A. Dobbs, M. Valtiner, and J. N. Israelachvili, Long-range electrostatic 
screening in ionic liquids, Proc. Natl. Acad. Sci. U. S. A. 112, 7432 (2015).

[31] Y. Jho, R. Brewster, S. A. Safran, and P. A. Pincus, Long-range interaction between heterogeneously charged membranes, Langmuir 27, 4439 (2011).

[32] B. Balu and A. S. Khair, Role of stefan-maxwell fluxes in the dynamics of concentrated electrolytes, Soft Matter 14, 8267 (2018).

[33] P. García-Sánchez, A. Ramos, A. González, N. G. Green, and H. Morgan, Flow reversal in traveling-wave electrokinetics: An analysis of forces due to ionic concentration gradients, Langmuir 25, 4988 (2009).

[34] C. L. Wirth, P. J. Sides, and D. C. Prieve, Electrolyte dependence of particle motion near an electrode during ac polarization, Phys. Rev. E 87, 032302 (2013).

[35] J. H. Wang, Self-Diffusion Coefficients of Water, J. Phys. Chem. 69, 4412 (1965).

[36] W. Warren and R. Cuthrell, Electrostatic forces between conducting spheres at constant potentials, J. Appl. Phys. 46, 4597 (1975).

[37] D. Jeffrey and M. Van Dyke, The temperature field or electric potential around two almost touching spheres, IMA J. Appl. Math. 22, 337 (1978).

[38] J. Lekner, Electrostatic force between two conducting spheres at constant potential difference, J. Appl. Phys. 111 (2012).

[39] B. Derjaguin, Untersuchungen über die reibung und adhäsion, iv, Colloid Polym. Sci. 69, 155 (1934).

[40] H. G. Weller, G. Tabor, H. Jasak, and C. Fureby, A tensorial approach to computational continuum mechanics using object-oriented techniques, Comput. Phys. 12, 620 (1998).

[41] H. Jasak, A. Jemcov, Z. Tukovic, et al., Openfoam: A c ++ library for complex physics simulations, in International workshop on coupled methods in numerical dynamics, Vol. 1000 (IUC Dubrovnik Croatia, 2007) pp. 1-20.

[42] R. Eymard, T. Gallouët, and R. Herbin, Finite volume methods, Handbook of Numerical Analysis 7, 713 (2000).

[43] H. K. Versteeg and W. Malalasekera, An Introduction to Computational Fluid Dynamics: the Finite Volume Method (Pearson education, 2007).

[44] J. A. Stratton, Electromagnetic Theory (John Wiley \& Sons, 2007).

[45] A. Castellanos, Electrohydrodynamics, Vol. 380 (Springer, 2014).

[46] J. N. Israelachvili, Intermolecular and Surface Forces (Academic press, 2011). 
[47] W. B. Russel, D. A. Saville, and W. R. Schowalter, Colloidal Dispersions (Cambridge university press, 1991).

[48] M. Z. Bazant, K. Thornton, and A. Ajdari, Diffuse-charge dynamics in electrochemical systems, Phys. Rev. E 70, 021506 (2004).

[49] S. Kim and S. J. Karrila, Microhydrodynamics: Principles and Selected Applications (Butterworth-Heinemann, London, 1991).

[50] R. E. Hansford, On converging solid spheres in a highly viscous fluid, Mathematika 17, 250 (1970).

[51] J. M. Crowley, Simple expressions for force and capacitance for a conductive sphere near a conductive wall, Proc. ESA Annu. Meet. Electrostatics (2008).

[52] U. Raviv, P. Laurat, and J. Klein, Fluidity of water confined to subnanometre films, Nature 413, $51(2001)$. 


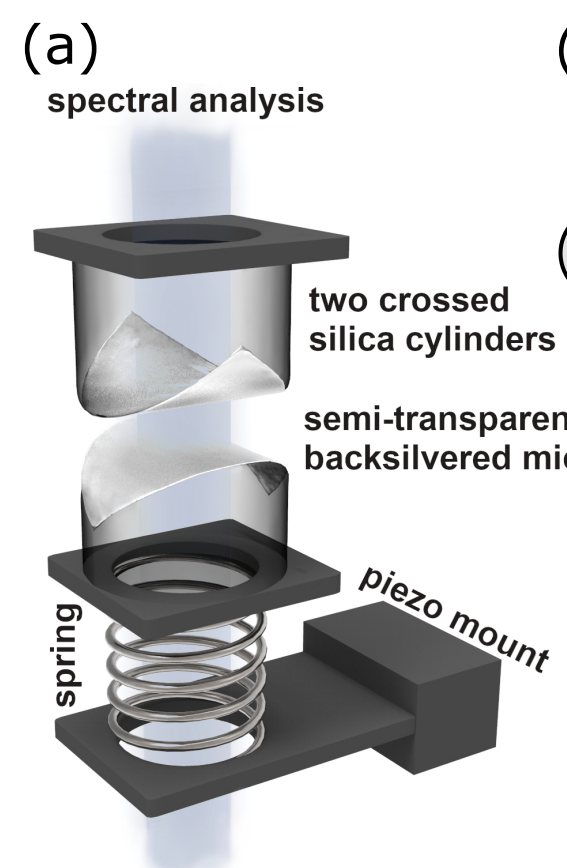

white light (b)

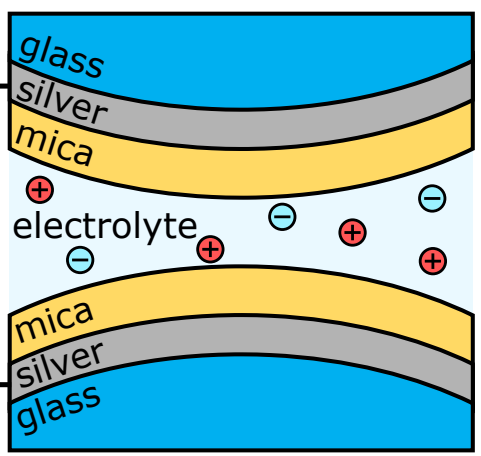

(d)

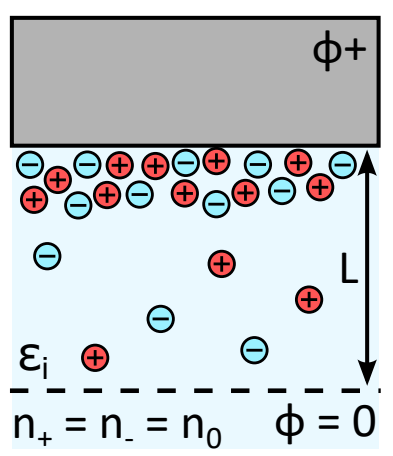

Fig. 1. Demonstration of the experimental system for the observed repulsion. (a) The surface forces apparatus (SFA) consisted of two back-silvered mica surfaces, aligned in a crossed-cylinder geometry and mounted on a spring and piezoelectric holder. (b) SFA capacitor. The space between the plates (silver layers) was filled with mica and electrolyte. Application of $\mathrm{AC}$ voltage of frequency between $20 \mathrm{~Hz}$ and $500 \mathrm{kHz}$ resulted in oppositely charged surfaces. (c) Measured surface separation upon application of the AC voltage 10 $\mathrm{V}$ and $100 \mathrm{kHz}$ with $1 \mathrm{mM} \mathrm{HNO}_{3}$. After application of the electric field the repulsive force appeared on a time scale of around 4 minutes. When the field was turned off, the system relaxed on a comparable time scale. The present effect was observed for every utilized electrolyte solution. (d) The geometry of the model. Reduction of the geometry from crossed cylinders to the 1D case with one boundary permeable to the ion flux (also see Ref. [25]). Application of an alternating electric field resulted in an excess of anions and cations in the vicinity of the solid electrode. 

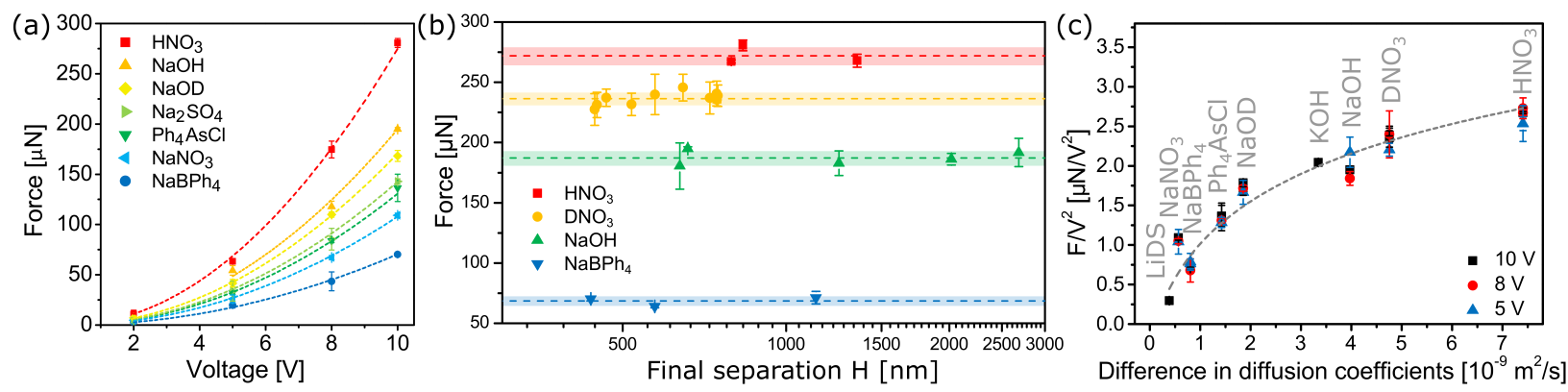

Fig. 2. Demonstration of the influence of the electrolyte and applied voltage. (a) The influence of different salts of $1 \mathrm{mM}$ concentration upon application of $100 \mathrm{kHz} \mathrm{AC}$ voltage. In every case, the observed repulsion scaled with the square of the applied voltage. Dashed lines corresponds to $F=A V^{2}$, where $A$ is a fitting parameter. (b) Influence of separation distance between the mica surfaces. The measured force was independent of the separation between the mica surfaces, which we explain by the fact that the electric field changed only $2.7 \%$ for the range of separations (Fig. S12 Ref. [25]). (c) Comparison of the measured repulsion (normalized by $V^{2}$ ) with magnitude of difference of diffusion coefficients $\left(\left|D_{+}-D_{-}\right|\right.$) of ions in various electrolyte solutions. 
(a)

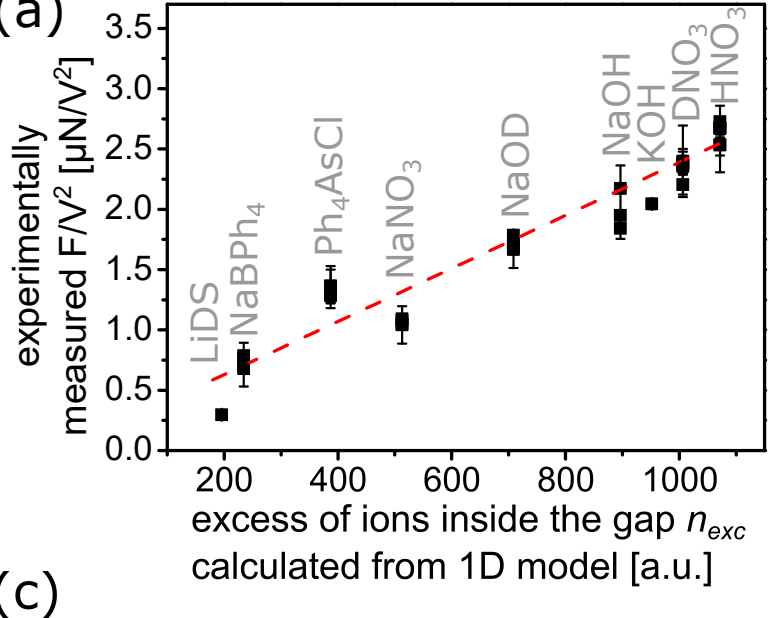

(c)

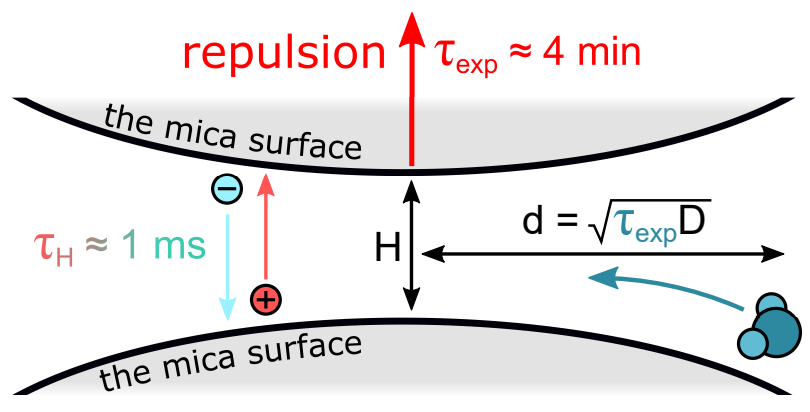

(b)

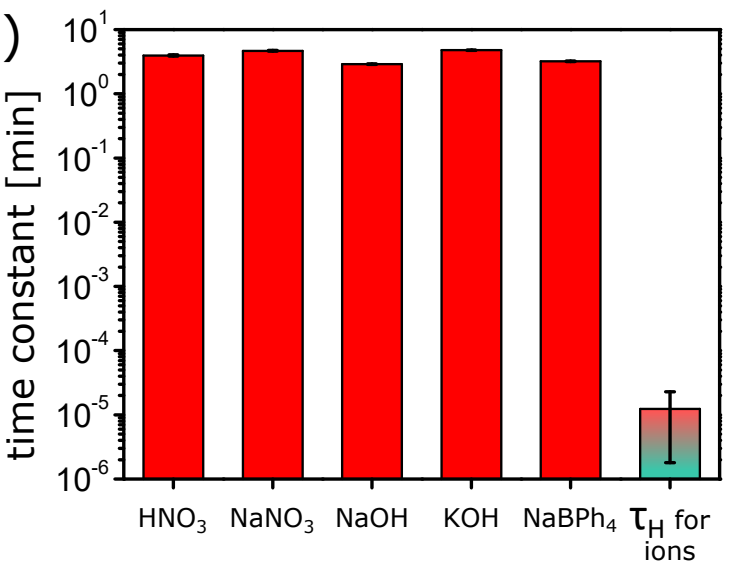

(d)

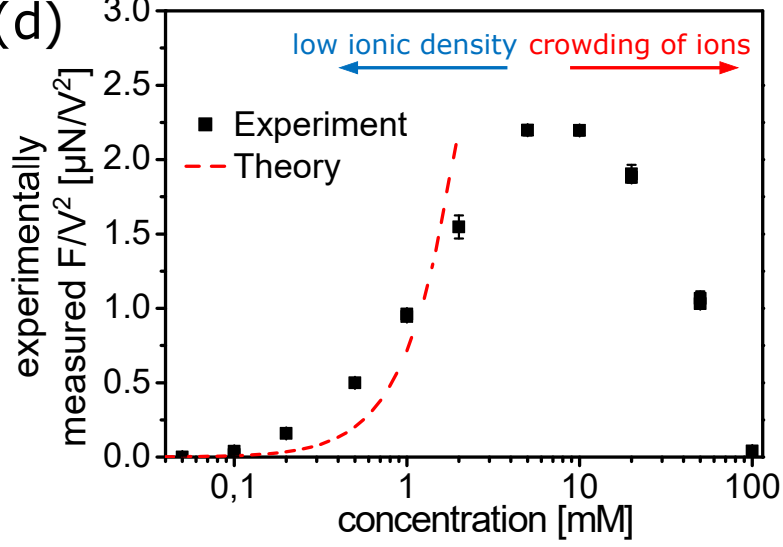

Fig. 3. Comparison of experiment and theory. (a) Comparison of theoretically predicted excess of ions $\left(n_{\text {exc }}\right)$ in the gap and experimentally measured force for $1 \mathrm{mM}$ solution upon application of $10 \mathrm{~V}$ and $100 \mathrm{kHz}$ AC voltage. The dashed line represents represents $F / V^{2}=B n_{\text {exc }}$. The correlation supports the idea that a strong repulsive force originates from the accumulation of ions, i.e., an increase of the osmotic pressure in the system. (b) Characteristic time scales for experiments with various electrolytes (see Fig. S2; Eq. 2 Ref. [25]). Experimental characteristic times of the process $\tau_{\text {exp }}$ (red bars) were similar and independent of the solution. Obtained times were much longer than average diffusive relaxation time across the narrow gap $\tau_{H}$ (red-blue bar, cf. Table S1 Ref. [25]). (c) Schematic of important time scales in the SFA system. During the $\tau_{\text {exp }}$ the solute and water diffuse over the horizontal dimension of the gap indicated as $d$. (d) Measured repulsive force for different concentrations of aqueous solution of $\mathrm{NaNO}_{3}$ upon application of $\mathrm{AC}$ voltage of $10 \mathrm{~V}$ and $100 \mathrm{kHz}$. Theory $\left(B n_{\text {exc }}\right.$ where $B$ is derived from panel (a)) predicts an increase of the force with increasing concentration of ions. The model does not include the finite sizes of ions, therefore does not capture the experimental trends at higher concentrations. The only free 
parameter in the 1D model is it's size $L=2 \mu \mathrm{m}$, which represents half the distance between the electrodes. 